\title{
Targeting the Hsp90-Cdc37-client protein interaction to disrupt Hsp90 chaperone machinery
}

\author{
Ting Li ${ }^{1}, \mathrm{Hu}$-Lin Jiang ${ }^{2}$, Yun-Guang Tong ${ }^{3,4}$ and Jin-Jian Lu ${ }^{1 *}$ (D)
}

\begin{abstract}
Heat shock protein 90 (Hsp90) is a critical molecular chaperone protein that regulates the folding, maturation, and stability of a wide variety of proteins. In recent years, the development of Hsp90-directed inhibitors has grown rapidly, and many of these inhibitors have entered clinical trials. In parallel, the functional dissection of the Hsp90 chaperone machinery has highlighted the activity disruption of Hsp90 co-chaperone as a potential target. With the roles of Hsp90 co-chaperones being elucidated, cell division cycle 37 (Cdc37), a ubiquitous co-chaperone of Hsp90 that directs the selective client proteins into the Hsp90 chaperone cycle, shows great promise. Moreover, the Hsp90-Cdc37-client interaction contributes to the regulation of cellular response and cellular growth and is more essential to tumor tissues than normal tissues. Herein, we discuss the current understanding of the clients of Hsp90-Cdc37, the interaction of Hsp90-Cdc37-client protein, and the therapeutic possibilities of targeting Hsp90-Cdc37-client protein interaction as a strategy to inhibit Hsp90 chaperone machinery to present new insights on alternative ways of inhibiting Hsp90 chaperone machinery.
\end{abstract}

Keywords: Hsp90 chaperone machinery, Cdc37, Kinase client, Protein interaction

\section{Background}

Heat shock protein 90 (Hsp90) is a critically conserved protein and one of the major molecular chaperones within eukaryotic cells [1]. It is the key component of the Hsp90 chaperone machinery, which facilitates protein folding and regulates the stabilization and activity of a large number of client proteins [2-4]. Hsp90 client proteins originate from distinct functional classes, including transcription factors (e.g., HIF1 $\alpha$, ATF3, and p53), steroid hormone receptors (e.g., estrogen receptor, glucocorticoid receptor, and progesterone receptor), and kinases (e.g., EGFR, B-raf, and SRC). Many of these client proteins are commonly overexpressed and/or frequently mutated in cancer cells [5-7]. Therefore, targeting Hsp90 offers a promising multitherapeutic strategy for cancer treatment.

Hsp90 family contains five subfamilies, which include cytosolic HSP90A, endoplasmic reticulum-localized HSP90B,

\footnotetext{
* Correspondence: jinjianlu@umac.mo; jinjian.lu@163.com

${ }^{1}$ State Key Laboratory of Quality Research in Chinese Medicine, Institute of Chinese Medical Sciences, University of Macau, Avenida da Universidade,

Taipa, Macau, China

Full list of author information is available at the end of the article
}

chloroplast HSP90C, mitochondrial TNFR-associated protein, and bacterial high-temperature protein $\mathrm{G}[2,8]$. In this review, we use the term Hsp90 to refer to these Hsp90 members when we do not explicitly distinguish the differences between them. Hsp90 functions as a homodimer, and each Hsp90 monomer comprises three critical conserved domains. The amino-terminal domain that is also referred to as $\mathrm{N}$-terminal domain mediates its ATP binding activity, the middle domain is the center of ATP hydrolysis and client binding, and the carboxy-terminal domain that is also referred to as the C-terminal domain is responsible for its dimerization [2, 9, 10]. ATP binding results in the rearrangement of the three domains, which induces a shift from an open conformation to a closed conformation [11]. The ATPase activity of Hsp90 is thought to be essential for the chaperone cycle of Hsp90 chaperone machinery [2]. Hsp90-directed agents that block ATP binding to Hsp90 have been established, such as geldanamycin (GM)-based inhibitors (GM, 17-AAG, 17-DMAG, and IPI-504), purine-based inhibitors (PU3, BIIBO21, PU-H71, MPC-3100, and CUDC-305), and radicicol-based inhibitors (NVP-AUY922, STA-9090, AT- 
13387, and KW-2478) [12-17]. Preclinical data have suggested Hsp90 as a potential anticancer target; however, the clinical trials have been halted or postponed in many cases. So far, none of the inhibitors for Hsp90 intervention have been approved for clinical application, which may be due to toxicity and heat shock response upon clinical treatment $[2,18,19]$. As a consequence, the strategy of blocking Hsp90's ATP-binding activity for the development of Hsp90 inhibitor is still under controversy.

Alongside the Hsp90 chaperone, co-chaperones are crucial regulators that drives the diverse functions of Hsp90 chaperone machinery. These co-chaperones, such as $\mathrm{Hsp} 70 / \mathrm{Hsp} 90$ organizing protein (HOP), cell division cycle 37 (Cdc37), small glutamine-rich TPR-containing protein alpha (SGTA), TPR repeat-containing protein associated with Hsp90 (TAH1), activator of Hsp90 ATPase protein 1 (AHA1), protein phosphatase 5 (PP5), FK506binding protein 5 (FKBP5), carboxyl terminus of HSC70interacting protein (CHIP), cyclophilin 40 (CYP40), p23, tetratricopeptide repeat domain 4 (TTC4), and uncoordinated mutant number 45 (Unc45), in concert with Hsp90 to form transient complexes not only mediate the Hsp90 ATPase cycle but also direct a broad range of specific clients to Hsp90 [2]. Among them, Cdc37, also known as p50, is one of the best-studied co-chaperones of Hsp90 [20-23] and proposed to be exclusively associated with protein kinases [24, 25]. Recent progress has allowed the interactions of Hsp90-Cdc37-client protein to be defined $[20,26,27]$. Herein, we discuss the current understanding of the clients of Hsp90-Cdc37, the interaction of Hsp90Cdc37-client, and the prospects of targeting Hsp90Cdc37-client interaction as a target for therapeutic intervention of the Hsp90 chaperone machinery.

\section{The client proteins of Hsp90-Cdc37}

Cdc37, first identified as a cell cycle protein in 1980 [28], was found to be an important intracellular cofactor of Hsp90 in 1981 [25, 29]. As a specific co-chaperone, Cdc37 is a selectively acquired recruiter for Hsp90 chaperone machinery to control the entry of diverse protein kinases [30]. The majority of kinase proteins interact with both Hsp90 and its co-chaperone Cdc37, and the Hsp90-mediated maturation of kinases is proposed to be strictly dependent on $\operatorname{Cdc} 37[20,26]$. To date, nearly 300 client proteins of Hsp90-Cdc37, which include diverse proteins such as EGFR, SRC, B-raf, AKT1, and CDK4, have been found [20,31]. To obtain a better understanding of the biological function and molecular interaction of the client proteins of Hsp90-Cdc37, a stand-alone software tool FunRich (version 3.0), which is mainly used for the functional enrichment and interaction network analysis, was applied [32]. A wellannotated list of Hsp90-Cdc37 client proteins was maintained and updated by the Picard laboratory, a research group that focus on the development of Hsp90 (see the online link: https://www.picard.ch/downloads/Cdc37in teractors.pdf) [31], and 267 proteins were successfully matched with the FunRich database (human only). Overall, our results demonstrated that the molecular functions of these proteins were mainly implicated in regulation of protein serine/threonine kinase activity (143 proteins), and the biological processes in which they mainly participated were cell communication (192 proteins) and signal transduction (214 proteins) (detailed data are shown in Additional file 1). These client proteins are involved in various biological pathways, such as the glypican pathway, IFN- $\gamma$ pathway, TRAIL signaling pathway, ERBB receptor signaling pathway, and VEGF/ VEGFR signaling network. Actually, most of the proteins do not operate alone but interact with each other and work in complexes while performing their functions in cellular progresses. The protein-protein interaction (PPI) plays a critical role in the signal transduction pathways and networks during diverse physiological processes [33]. In the present study, the PPI network visualization and its analysis were also performed, and 449 PPI pairs were formed by the 267 different client proteins (Fig. 1a). Among them, 29 client proteins, including SRC, EGFR, FYN, Raf-1, and AKT1, formed at least 10 pairs in the PPI network and were defined as core client proteins (Table 1). Different EGFR family members with various biological activities could form the ligandtriggered heterodimers with family members, resulting in activation of complex signals [34-36]. IGF1R was also identified to form heterodimers with insulin receptor (gene symbol: IRSNN) and EGFR family members, conferring resistance to homo-therapy [37, 38]. The Raf-1/B-rafformed heterodimers exhibit highly increased kinase activity than the respective homodimers [39]. Clearly, an individual protein alone cannot be a simple predictor of drug efficiency; functional protein-protein dimers with distinct pharmacological properties are also required [40-44]. Herein, the complex protein network and sundry PPI pairs formed by these Hsp90-Cdc37 clients in turn heightened the understanding of Cdc37 as a key factor in Hsp90 chaperone machinery.

\section{The interaction of Hsp90-Cdc37-client protein}

Various studies in recent years have helped determine how Cdc37 enables Hsp90 chaperone machinery to recognize specific client proteins progressively; however, the full-length structures of client protein with either Hsp90 or Cdc37 remain to be elucidated [1, 2, 27, 45]. Similar to Hsp90, Cdc37 contains three domains (N-terminal domain, M-domain, and the C-terminal domain) that function cooperatively [26, 27]. Cdc37 acts as a structural defect discriminator and a kinase sorting module in the Hsp90 chaperone machinery [46]. As 


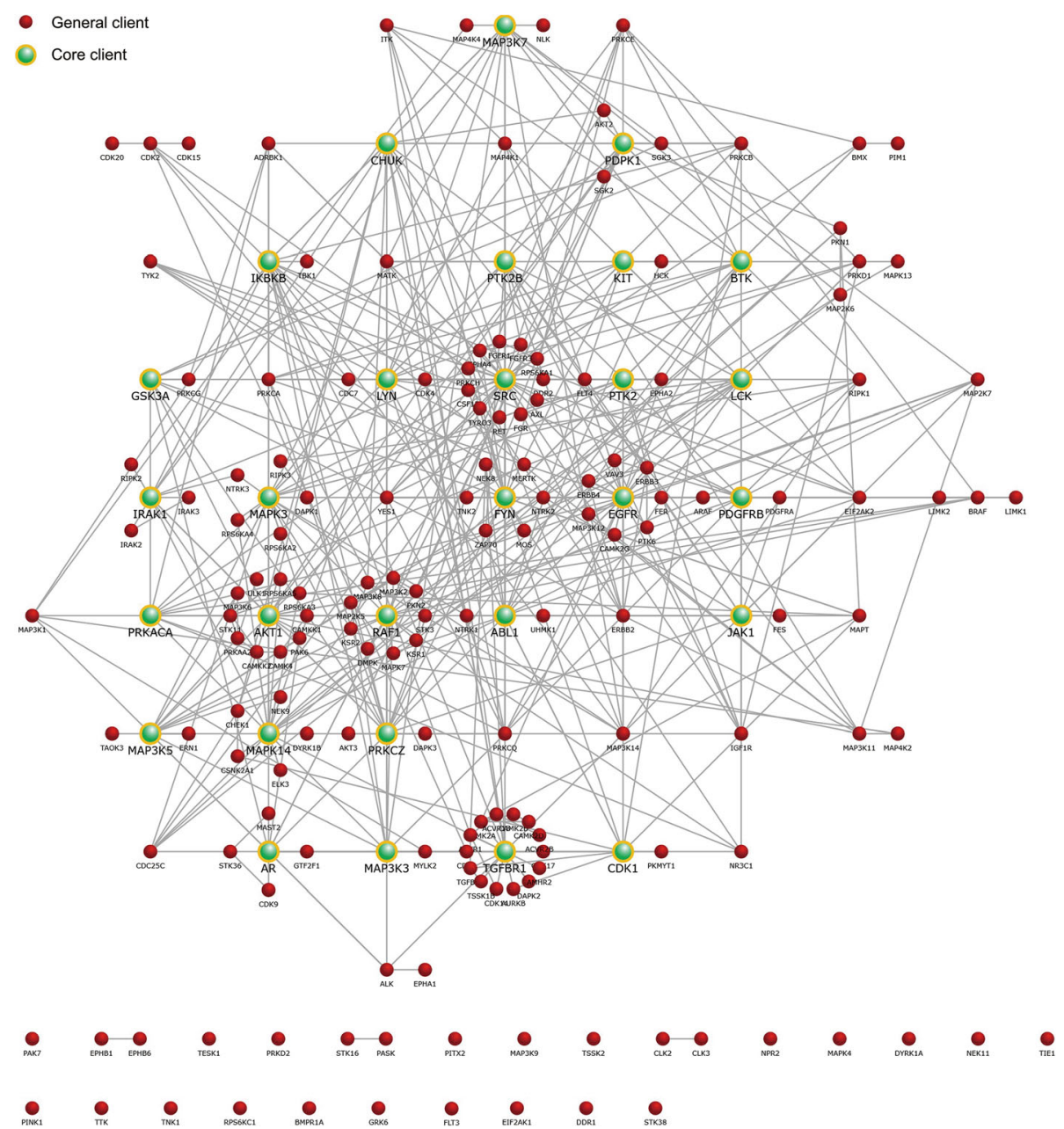

Fig. 1 The protein-protein interaction of Hsp90-Cdc37's client proteins. Using the FunRich tool to analyze the protein-protein interaction of Hsp90Cdc37's client proteins. 449 PPI pairs are formed by these 267 proteins in total. PPI: protein-protein interaction

shown in Fig. 2, Cdc37 first tests the proper substrates and establishes stable connection with the client protein to create a Cdc37-client protein binary complex. Then, the binary complex binds to Hsp90 to form a ternary complex. The formation of the Hsp90-Cdc37-client protein ternary complex finally facilitates client protein loading onto the Hsp90 chaperone machinery.

Of note, Cdc37 will be phosphorylated by casein kinase 2 (CK2) at Ser13 before connecting with client proteins [47] and dephosphorylated by the protein phosphatase PP5 before client protein release [48]. Proper phosphoserine of Cdc37 at Ser13 is necessary for Hsp90 chaperone machinery to recognize the structural perturbation at the client protein domain and helps Cdc37 to stabilize its own structure and participate in the interaction with Hsp90 [27]. These works have heightened the understanding of the phosphorylation on Cdc37's conformation and ability to bind to Hsp90. Besides phospho-Ser13 of Cdc37, previous studies have recognized GSGSFG, a glycine-rich motif, as a unique sequence that is necessary for the stable client protein's interaction with $\mathrm{Cdc} 37$ [49, 50], and the adjoining loop linking the $\alpha$-C-helix to the $\beta 4$-strand and the $\alpha$-Chelix of the client protein also appears to be the primary determinant to be recognized by Cdc37 [51]. The difference of C-terminal portions within client proteins takes part in determining the sharp affinity activity with Cdc37, which may explain the different affinities between CDK4 (Cdc37dependent protein) and CDK2 (non-Cdc37-dependent protein) despite the similar glycine-rich loop in the $\mathrm{N}$-terminal portions [49]. Nevertheless, based on the analysis using FunRich tool, more than $50 \%(P<0.001)$ of the client proteins contain the serine/threonine kinase catalytic domain (S-TKc), suggesting that this kind of domain may be preferable than others. Even though the definitive features of Cdc37 and client proteins have not yet been clearly illustrated, several key portions are crucial for the physical association of client protein and Cdc37 and determine the triage process regarding Cdc37's dependence. 
Table 1 The core client proteins. The 29 client proteins formed at least 10 pairs in the PPI network

\begin{tabular}{|c|c|c|c|}
\hline Item & Protein name & Gene symbol & Molecular functions \\
\hline 1 & SRC & SRC & Kinase, tyrosine-protein kinase, transferase \\
\hline 2 & EGFR & EGFR & $\begin{array}{l}\text { Developmental protein, host cell receptor for } \\
\text { virus entry, kinase, receptor, tyrosine-protein } \\
\text { kinase, transferase }\end{array}$ \\
\hline 3 & FYN & FYN & $\begin{array}{l}\text { Developmental protein, kinase, tyrosine-protein } \\
\text { kinase, transferase }\end{array}$ \\
\hline 4 & Raf-1 & RAF1 & Kinase, serine/threonine-protein kinase, transferase \\
\hline 5 & AKT1 & AKT1 & Kinase, serine/threonine-protein kinase, transferase \\
\hline 6 & ERK1 & MAPK3 & Kinase, serine/threonine-protein kinase, transferase \\
\hline 7 & P38 & MAPK14 & Kinase, serine/threonine-protein kinase, transferase \\
\hline 8 & FAK1 & PTK2 & Kinase, tyrosine-protein kinase, transferase \\
\hline 9 & IKK- $\beta$ & IKBKB & Kinase, serine/threonine-protein kinase, transferase \\
\hline
\end{tabular}

\begin{tabular}{|c|c|c|}
\hline TGFR-1 & TGFBR1 & $\begin{array}{l}\text { Kinase, receptor, serine/threonine-protein kinase, } \\
\text { transferase }\end{array}$ \\
\hline LYN & LYN & Kinase, tyrosine-protein kinase, transferase \\
\hline PKC2 & PRKCZ & Kinase, serine/threonine-protein kinase, transferase \\
\hline PDK1 & PDPK1 & $\begin{array}{l}\text { Activator, kinase, serine/threonine-protein kinase, } \\
\text { transferase }\end{array}$ \\
\hline IKK-a & CHUK & Kinase, serine/threonine-protein kinase, transferase \\
\hline
\end{tabular}

15 MEKK7 MAP3K7 Kinase, serine/threonine-protein kinase, transferase

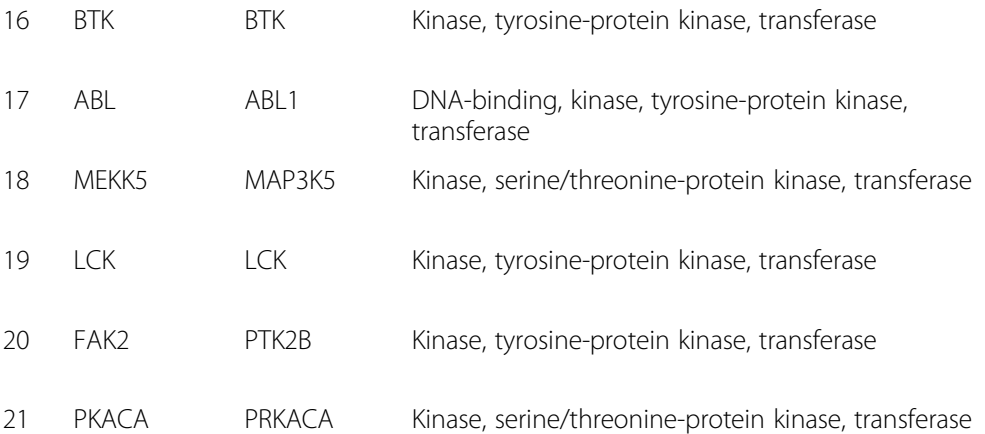

Partner of PPI pair

MAP4K1, PRKCA, PRKCE, RET, TYRO3, PTK2B, CHUK,

DDR2, ADRBK1, EGFR, BMX, KIT, PDGFRB, RAF1, ERBB2, IKBKB, MATK, PRKCZ, ABL1, PTK2, AXL, AR, PRKACA, CDC25C, AKT1, ITK, FYN, IGF1R, CSF1R, PRKCH, PDPK1, EPHA4, LYN, PRKD1, FGR

MAP4K1, EPHA2, AR, LYN, PDGFRB, PRKD1, FER, SRC, PTK2B, ERBB3, MAP3K12, CDK1, YES1, ERBB2, PRKCA, IGF1R, PTK2, RIPK1, MAP3K14, CAMK2A, ERBB4, VAV3, PTK6, ADRBK1, TNK2, PRKACA, CAMK2G, MAPK14

ITK, NTRK2, RAF1, NR3C1, ZAP70, TNK2, PRKCQ, PRKCE, TYK2, PTK2B, MAP4K1, CSF1R, TYRO3, PTK2, EPHA4, SRC, CDK1, YES1, PDGFRB, ABL1, PRKCZ, MAPT, NEK8, KIT, BTK, MOS, PRKCH

MAP3K1, PRKCZ, FYN, PDGFRB, AR, STK3, BRAF, SRC, KSR1, AKT1, PRKCA, JAK1, MAPK3, PRKCE, MAPK7, DMPK, PRKACA, MAP3K5, NR3C1, LCK, KSR2

PDPK1, PRKCQ, PKN2, AR, CHUK, MAP3K8, RAF1, BRAF, MAP3K11, PRKCZ, IKBKB, SRC, MAPK14, CAMKK1, AKT2, IRAK1, PAK6, MAP3K5, GSK3A, CHEK1

DAPK1, RPS6KA2, RPS6KA3, RPS6KA4, MAPK14, PRKCZ, RPS6KA1, KSR2, PRKCE, LCK, RAF1, CDC25C, MAP3K14, NTRK1, LYN, NTRK3, ADRBK1, BRAF, RET

IRAK1, DYRK1B, RPS6KA4, RPS6KA3, MAPK3, MAP3K7, CSNK2A1, TGFBR1, MAP2K6, MAP2K7, CAMKK2, AKT1, RPS6KA5, CDC25C, PKN1, ELK3, RET, EGFR

EPHA2, LYN, BMX, RET, IGF1R, SRC, EGFR, FLT4, FYN, FGR, PDGFRB, ERBB2, YES1, LCK, PTK2B, ERBB3, RIPK1

CHUK, MAP3K7, EIF2AK2, MAP3K14, MAP3K1, PRKCQ, PRKCB, MAP3K3, PRKCZ, SRC, TGFBR1, MAP3K11, AKT1, ACVR1, IRAK1, TBK1

CHUK, CDK17, AMHR2, DAPK2, AURKB, TGFBR2, MAPK14, CDK14, CDK6, IKBKB, MAP3K7, TSSK1B, NEK8, ITK, ACVR1, CDK4

TYK2, PTK2, MATK, EGFR, KIT, CDK2, CDK1, BTK, CDK4, CSF1R, PTK2B, PRKCQ, MAPK3, MAP4K1, SRC, MAP3K3

RAF1, DAPK3, MAPK3, JAK1, PDPK1, IKBKB, SRC, PRKCQ, AKT3, AKT1, GSK3A, MAP2K5, IRAK1, BTK, FYN, PRKCA

PKN1, AKT1, PRKACA, PKN2, RPS6KA1, RPS6KA3, PRKCZ, PTK2B, PRKCE, SGK3, AKT2, PRKCB, SRC, SGK2, AKT3

MAP3K8, MAP3K7, IKBKB, TGFBR1, SRC, MAP3K14, AKT1, AKT2, IRAK1, PRKCB, EIF2AK2, MAP3K11, PRKCQ, CHEK1, MAP3K3

CHUK, IKBKB, MAP4K1, MAP2K7, MAPK14, MAP2K6, MAP3K5, RIPK1, NLK, TGFBR1, MAP3K14, MAP3K3, EIF2AK2, MAP4K4, IRAK1

PRKCQ, PRKD1, LYN, JAK1, ABL1, BMX, IRAK1, PRKCE, PRKCZ, PRKCB, HCK, FYN, KIT, ITK, PRKCA

HCK, MAP4K1, PRKD1, SRC, NTRK1, NEK8, KIT, BTK, JAK1, ZAP70, CDK1, FYN, MAPT, UHMK1

MAP2K7, EIF2AK2, MAP3K6, MAP3K7, MAP2K6, MAP3K2, MAP3K3, PRKAA2, RAF1, ERN1, AKT1, IGF1R, ALK

PRKCA, ZAP70, ITK, PRKCQ, MAPK3, KIT, CDC25C, PRKACA, PTK2B, AXL, PTK2, RAF1, NR3C1

SRC, ZAP70, EGFR, FYN, PDPK1, ERBB3, JAK1, LYN, MATK, LCK, ERBB2, PTK2, FGFR3

PDPK1, STK11, NR3C1, SRC, GSK3A, CAMKK2, LCK, ADRBK1, RAF1, MAP3K3, EGFR, BRAF 
Table 1 The core client proteins. The 29 client proteins formed at least 10 pairs in the PPI network (Continued)

\begin{tabular}{|c|c|c|c|c|}
\hline Item & Protein name & Gene symbol & Molecular functions & Partner of PPI pair \\
\hline 22 & IRAK & IRAK1 & Kinase, serine/threonine-protein kinase, transferase & $\begin{array}{l}\text { MAPK14, CHUK, IRAK3, IRAK2, PRKCZ, MAP3K7, BTK, } \\
\text { AKT1, RIPK2, CAMKK2, IKBKB, NTRK3 }\end{array}$ \\
\hline 23 & JAK1 & JAK1 & Kinase, tyrosine-protein kinase, transferase & $\begin{array}{l}\text { IGF1R, PRKCZ, TYK2, RAF1, PTK2B, EIF2AK2, BTK, ABL1, } \\
\text { PDGFRA, FES, PDGFRB, FER }\end{array}$ \\
\hline 24 & CDK1 & CDK1 & $\begin{array}{l}\text { Host cell receptor for virus entry, kinase, receptor, } \\
\text { serine/threonine-protein kinase, transferase }\end{array}$ & $\begin{array}{l}\text { PKMYT1, CDC25C, LYN, EGFR, CSNK2A1, AR, PRKCB, } \\
\text { MAPT, FYN, ABL1, AURKB, TGFBR2 }\end{array}$ \\
\hline 25 & PDGFR-1 & PDGFRB & $\begin{array}{l}\text { Developmental protein, kinase, receptor, tyrosine- } \\
\text { protein kinase, transferase }\end{array}$ & $\begin{array}{l}\text { VAV3, RAF1, EGFR, SRC, PDGFRA, TYK2, ARAF, FYN, YES1, } \\
\text { EIF2AK2, JAK1, PTK2 }\end{array}$ \\
\hline 26 & MEKK3 & MAP3K3 & Kinase, serine/threonine-protein kinase, transferase & $\begin{array}{l}\text { MAP3K2, ALK, MAP2K5, IKBKB, MAP3K7, MYLK2, } \\
\text { MAP3K5, PRKACA, RIPK1, CHUK, LYN }\end{array}$ \\
\hline 27 & CD117 & $\mathrm{KIT}$ & Kinase, receptor, tyrosine-protein kinase, transferase & $\begin{array}{l}\text { LYN, SRC, MATK, HCK, YES1, LCK, ABL1, FYN, BTK, PRKCA } \\
\text { PRKCB }\end{array}$ \\
\hline 28 & $A R$ & AR & Activator, DNA-binding, receptor & $\begin{array}{l}\text { PAK6, AKT1, EGFR, GTF2F1, RAF1, GSK3A, NR3C1, CDK6, } \\
\text { SRC, CDK1, CDK9 }\end{array}$ \\
\hline 29 & GSK3a & GSK3A & $\begin{array}{l}\text { Kinase, serine/threonine-protein kinase, signal } \\
\text { transduction inhibitor, transferase }\end{array}$ & $\begin{array}{l}\text { PRKCA, AR, MAPT, PRKACA, PRKCZ, SGK3, MAP3K11, } \\
\text { PRKCB, AKT1, PRKCG }\end{array}$ \\
\hline
\end{tabular}

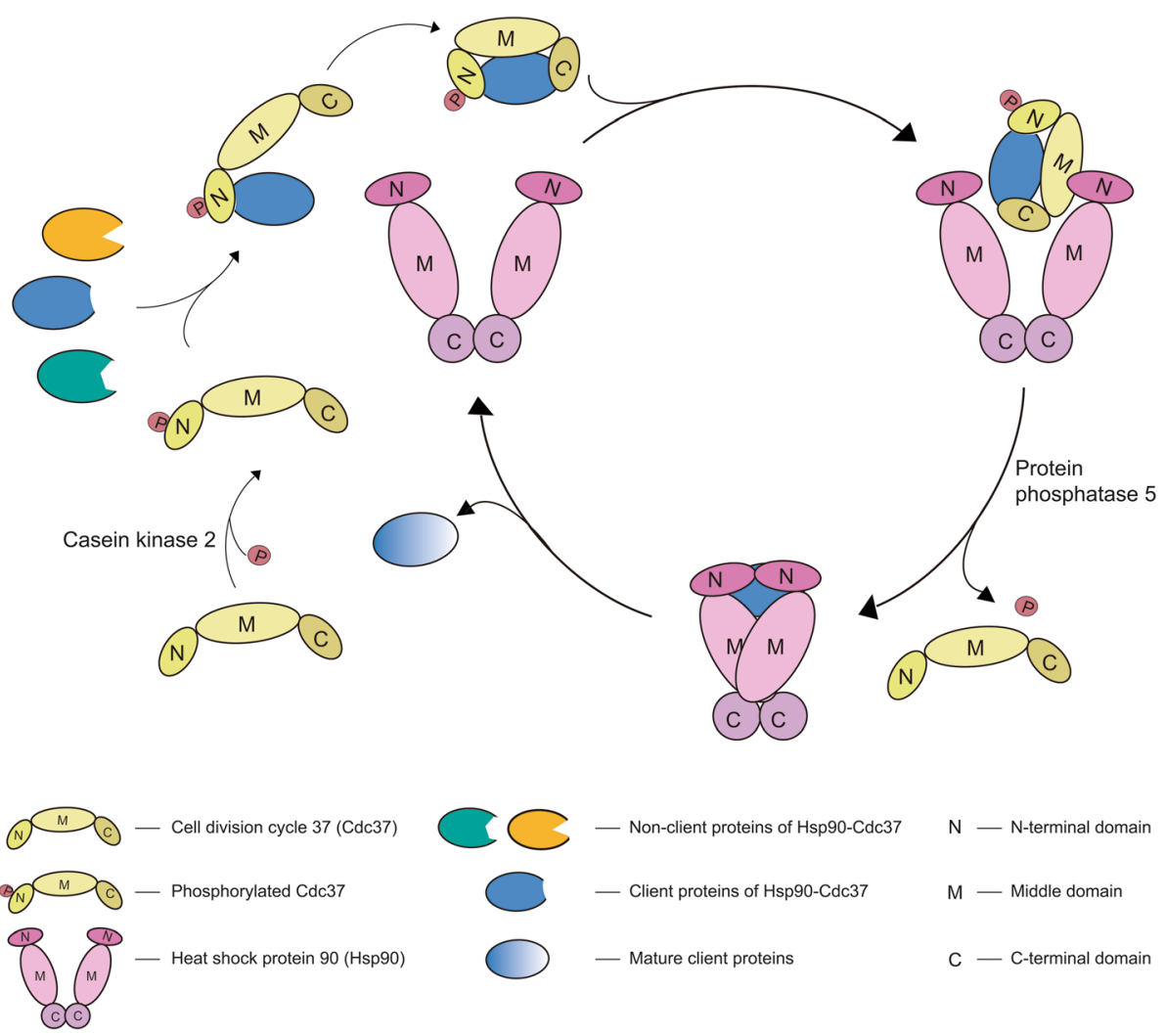

Fig. 2 A speculative model for the Hsp90-Cdc37-client protein cycle. Cdc37 first tests the proper substrates and establishes stable connection with the client protein to create a Cdc37-client protein binary complex. Then, the binary complex binds to Hsp90 to form a ternary complex. The formation of the Hsp90-Cdc37-client protein ternary complex finally facilitates client protein loading onto the Hsp90 chaperone machinery. It is worthy of note that $\mathrm{Cdc} 37$ will be phosphorylated by casein kinase 2 (CK2) at Ser13 before connecting with client proteins and dephosphorylated by the protein phosphatase 5 (PP5) before client protein release 
As for the binding site of Cdc37 and Hsp90, the classic one in humans is the middle domain of $\mathrm{Cdc} 37$ bond to the N-terminal domain of Hsp90 (shown in Fig. 2). Cdc37 uses the $\mathrm{N}$-terminal domain to test the proper substrates and applies its C-terminal region to build the stable connection with the client protein. The Cdc37-client protein complex will then bind to the $\mathrm{N}$-terminal domain of Hsp90 via the M-domain of Cdc37 [1, 2, 27, 45]. Specifically, the residues $127-163$ within the middle domain of Cdc37 were recognized as the inter-domain switch to sense the proper conformation of Hsp90 and regulate the binding activity of Cdc37-client protein complex with Hsp90 [52]. In addition, a second interaction site of Hsp90 for Cdc37 within Caenorhabditis elegans (CeCdc37) was uncovered recently, which is regulated by the binding of $\mathrm{N}$-terminal domain of $\mathrm{CeCdc} 37$ to the middle domain of Hsp90 [30, 53]. These two interactions utilized by $\mathrm{Cdc} 37$ within different species seem to function relevantly and mediate the conformational change and the ATPase activity of Hsp90.

Most recently, the client protein within the Hsp90Cdc37-client protein ternary complex was found to bind to the separated sides of Hsp90 using its two elongated and non-native lobes [27]. This structure suggested that the client protein remains in an uncompleted folded status even after the conformation of the Hsp90-Cdc37client protein ternary complex, and it still relies on the subsequent function of Hsp90 to reach maturity. This finding provides further evidence for the heavy dependence of client protein maturation on the cooperation of Cdc37 and Hsp90.

\section{Targeting Hsp90-Cdc37-client protein interaction to block} Hsp90 chaperone machinery

As the majority of kinases are adversely affected by Hsp90-Cdc37, drug design targeting Hsp90-Cdc37-client protein interaction has been highlighted as a promising novel strategy. Investigating technically feasible methods to modulate Hsp90-Cdc37 activity is of considerable importance. Based on the nature of Hsp90-Cdc37-client protein interaction, there are three potential categories that are likely to disrupt the function of Hsp90 chaperone machinery: targeting Cdc37, Cdc37-client protein interaction, and Hsp90-Cdc37 interaction.

\section{Targeting Cdc37}

Cdc37 has an increased level in proliferating tissues and organs and is highly expressed in certain tumors, such as prostate cancer [54]. Therefore, the tumor cells, rather than normal cells, have increasing dependency on the Cdc37 level. Moreover, as the recognizer of client protein being tied to the Hsp90 chaperone system, Cdc37 is primarily and specifically interacted with the kinase protein, whereas Hsp90 is widely associated with many classes of client proteins (transcription factors, steroid hormone receptors, and kinases) [55]. The absence of Cdc37 can only disrupt the interactions with kinase clients but not the interactions with non-kinase clients [20, 56]. Cdc37 is needed for both maturation and activation of client proteins, which may be dependent on the differences of species and tissue. Silencing Cdc37 using shRNA disrupts the Hsp90 chaperone machinery via impairing the association of client protein with $\mathrm{Hsp} 90$ and prevents protein maturation, which may subsequently induce a proteasomal degradation of client proteins, and finally suppresses the proliferation of human colon cancer cells [56]. Additionally, depletion of Cdc37 could also block the activities of many client proteins, instead of global degradation of client proteins. This blockage resulted in the suppression of multiple pathways (e.g., MAPKs and androgen-induced pathways) and induced growth inhibition in human prostate cancer cells [57]. Given the oncogenic role of many of these client proteins (e.g., EGFR, SRC, and Raf-1), this specificity of Cdc37, rather than that of Hsp90, provides a potential and available therapeutic window for Cdc37-targeted therapy. The current studies of targeting Cdc37 were mainly focused on the application of the relevant gene silencers (e.g., shRNA/siRNA). With the development of microRNA for protein mediation [58], regulating microRNA targeting Cdc37 to decrease Cedc37 should also be taken into consideration.

\section{Targeting Cdc37-client interaction}

As mentioned above, phosphorylated $\mathrm{Cdc} 37$ at the conserved Ser13 site is a prerequisite for the efficient binding activity of $\mathrm{Cdc} 37$ to protein kinases and crucial for the recruitment of the protein kinase-Cdc37 complex to Hsp90 [47, 59, 60]. The phosphorylation of Cdc37 (Ser13) is mediated by CK2 [61, 62]. Specific inhibition of CK2 with its chemical inhibitor, 4,5,6,7-tetrabromobenzotriazole, could reduce the phosphorylation of Cdc37 and the protein levels of Cdc37-dependent protein kinases [59]. Besides the phosphorylation, the dephosphorylation of Cdc37 is pivotal for its activation as well. PP5, a serine/threonine-protein phosphatase that regulates hormone- and stress-induced cellular signaling [48, 63, 64], mediates Cdc37 dephosphorylation and is critically dependent for client protein release. Dephosphorylation is also important for the reversal of Cdc37's resistance to nonspecific phosphatases and constitutive phosphorylation $[48,65]$. These results show the regulatory mechanisms of Cdc37 activity and the molecular basis for the ability of CK2 and PP5 to regulate cellular functions of the Cdc37-client protein complex. Moreover, the finding that the phosphatase ability of PP5 is active only when it is interacting with proteins (e.g., Hsp90) via its TPR domain [66] has provided further 
evidence for targeting PP5 as a relatively specific means of blocking Hsp90 chaperone machinery.

Specific ATP-competitive kinase inhibitors may be another kind of candidates that block Cdc37 interaction with protein kinases. The kinase inhibitors (e.g., the Braf inhibitor vemurafenib and the EGFR/HER2 inhibitor lapatinib) were found to antagonize $\mathrm{Cdc} 37$ interaction with target kinases not only by inducing the chaperone deprivation of nascent protein but also by disrupting the formed Hsp90-Cdc37-kinase complex. This Cdc37 antagonism finally leads to kinase depletion in a manner similar to that of Hsp90 inhibitor. Thus, the kinase inhibitors, besides the role that they are meant to play, also seem to act as antagonists of PPI within the Hsp90 molecular chaperone machinery [67]. These results also suggest that in some dependent tumor cells, Cdc37 chaperone deprivation, at least in part, contributes to the efficiency of targeted kinase inhibitors.

\section{Targeting Hsp90-Cdc37 interaction}

During the past few years, several kinds of disruptors toward the association of Hsp90 and Cdc37 have been discovered, as shown in Table 2. The underlying mechanisms of this kind of compounds mainly focus on the replacement of key residue-residue interaction between Hsp90 and Cdc37. Most of these disruptors are natural products, such as celastrol [68, 69], sulforaphane [70], FW-04-806
[71, 72], withaferin A [73-75], kongensin A [76, 77], and platycodin $\mathrm{D}[78,79]$. In addition to the small molecules, the peptide-derived disruptors also offer another way to block the interaction of Hsp90-Cdc37. For instance, the developed Cdc37-derived peptide Pep-1 exhibited competitive binding affinity to $\mathrm{Hsp} 90$ at the micromolar level though occupying the Cdc37 binding site. This binding subsequently leads to the disruption of Hsp90-Cdc37 PPI and the ATP bond to Hsp90 [80].

\section{Conclusion}

The Hsp90 chaperone machinery is known to be a multiprotein complex, which includes the chaperone (Hsp90) and co-chaperones (e.g., HOP, Cdc37, and AHA1). This chaperone machinery has been regarded as prime importance to cancer survival with its effort on assistance of general protein folding and prevention of protein misfolded or unfolded actions. Hsp90, the famous heat shock protein, is the key factor for this chaperone machinery and allows cancer cells to adapt to severe stress [55, 81]. In the past decade, tremendous efforts have been made to develop Hsp90 inhibitors as anticancer agents [2, 82, 83]. However, none of the directed Hsp90 inhibitors have achieved the goal and have been applied in clinical treatment $[2,18,19]$. Seeking for another alternative strategy to inhibit the Hsp90 chaperone machinery and achieve better therapeutic efficiency is thus urgent.

Table 2 Targeting the Hsp90-Cdc37-client protein interaction to disrupt Hsp90 chaperone machinery

\begin{tabular}{|c|c|c|}
\hline $\begin{array}{l}\text { The potential approaches to disrupt the } \\
\text { Hsp90-Cdc37-client protein interaction }\end{array}$ & Related mechanisms & References \\
\hline \multicolumn{3}{|l|}{ Targeting Cdc37 } \\
\hline siRNAs or shRNA & Silencing Cdc37 & {$[56,57]$} \\
\hline \multicolumn{3}{|l|}{ Targeting Cdc37-client interaction } \\
\hline 4,5,6,7-Tetrabromobenzotriazole & CK2 inhibitors, suppressing Cdc37 phosphorylation at Ser13 site & [59] \\
\hline PP5 mutation & Suppressing Cdc37 dephosphorylation & [48] \\
\hline Vemurafenib & B-raf inhibitor. Antagonize Cdc37 interaction with kinases & [67] \\
\hline Lapatinib & EGFR/HER2 inhibitor. Antagonize Cdc37 interaction with kinases & [67] \\
\hline \multicolumn{3}{|l|}{ Targeting Hsp90-Cdc37 interaction } \\
\hline Celastrol & Blocking the critical interaction of $\mathrm{Hsp} 90$ at Glu33 and Cdc37 at Arg167 & {$[68,69]$} \\
\hline Sulforaphane & $\begin{array}{l}\text { Disrupting the formation of Hsp90-Cdc37 complex by direct modification } \\
\text { of specific amino acids residues of Hsp90 }\end{array}$ & [70] \\
\hline FW-04-804 & Binding sites at Gln133 and Glu47/Arg46 of Hsp90 & {$[71,72]$} \\
\hline Withaferin A & $\begin{array}{l}\text { Blocking several H-bond between Hsp90-Cdc37 interaction, like Ser113 } \\
\text { (Hsp90)-Gln208 (Cdc37) bond and Gln133 (Hsp90)-Arg166 (Cdc37) bond }\end{array}$ & [73-75] \\
\hline Kongensin A & $\begin{array}{l}\text { Covalently binds to a cysteine } 420 \text { in the middle domain of Hsp90 and } \\
\text { dissociates Hsp90 from Cdc37 }\end{array}$ & {$[76,77]$} \\
\hline Platycodin D & $\begin{array}{l}\text { H-bond connection with Hsp90 at Arg32 and Phe200 and Cdc37 at } \\
\text { Asp169 and Asp170 }\end{array}$ & {$[78,79]$} \\
\hline Pep-1 & $\begin{array}{l}\text { Cdc37-derived peptides, bound to Hsp90 N-terminal domain and inhibited } \\
\text { Hsp90 ATPase activity }\end{array}$ & [80] \\
\hline
\end{tabular}

There are three potential approaches to disrupt the function of Hsp90 chaperone machinery: targeting Cdc37, Cdc37-client protein interaction, and Hsp90-Cdc37 interaction 
Being the crucial recognizer of protein kinases to tie to the Hsp90 system, Cdc37 may be a promising candidate target. Most of the client proteins of $\mathrm{Hsp} 90-\mathrm{Cdc} 37$ are highly involved in key signal transduction systems and regulate cellular activities [84-86]. Besides the well-known client proteins of Hsp90-Cdc37 (e.g., HER2, CDK4, CDK6, and AKT), the key signaling molecules of necroptosis, the activation of receptor-interacting protein kinases 1-3 (RIP1-3), were found to be dependent on the interaction with the Hsp90-Cdc37 complex [76, 87, 88], which may help to generate a new approach against tumors with apoptotic deficiency. Androgen receptor (AR) is not a kinase protein but is recognized as a specific client protein of Hsp90-Cdc37 [89]. Given the key role of AR for transcriptional mediation in prostate cancer, the feasibility of targeting Hsp90-Cdc37 activity to mediate AR may be useful for prostate cancer treatment. Nevertheless, the resistance to directed Hsp90 inhibitors could also be reversed by silencing Cdc37 via destabilization of client proteins. This evidence supports that the blockage of Hsp90-Cdc37client protein interaction can be an effective target for discovering novel inhibitors toward Hsp90 chaperone machinery and shows the potential to be applied in cancer treatment.

To date, several approaches are proven useful in blocking the association of Hsp90-Cdc37-client protein (as shown in Table 2); however, challenges remain. Firstly, the application of Cdc37-targeted intervention has not been fulfilled in vivo. To improve the stabilization and the transfection efficiency of RNA interference-Cdc37 [90] and to figure out the clear microRNA that targets Cdc37 will have superior therapeutic effect. Secondly, although the CK2 and PP5 are required for efficient phosphorylation and dephosphorylation of Cdc37, the CK2- or PP5-targeted therapies for clinical application are still under investigation [91]. Thirdly, the reported inhibitors of the Hsp90-Cdc37 complex so far are mainly natural products with poor selectivity, which may limit their potency to be drug candidates. The basic research of the Cdc37-derived peptides is still ongoing [92]. The structure modification of the current agents may provide further pharmacological properties of current agents while retaining target specificity of Hsp90-Cdc37 complex.

More still needs to be explored regarding the crucial role of Hsp90-Cdc37-client protein interaction and its effectiveness as target for cancer treatment. As mentioned above, depletion of Cdc37 may lead to the decrease of cell survival signals through either the degradation of the client protein level or the inhibition of client protein activity [56, 57]. The inhibition of Hsp90-Cdc37 interaction by the platycodin D also caused distinct regulation of client proteins as demonstrated by decreased activity and conserved protein level of AKT [78, 93]. Thus we remain uncertain of the full information of cancer types mediated by the regulation of Hsp90-Cdc37-client protein interaction. In addition, studies indicated the dysregulation of some client proteins obviously prefers certain cancer to other normal tissues. As AR is essential for the development of prostate cancer, and EGFR is a key factor for definite lung cancer, future research will be indispensable to pay attention to the stratification of patients before the application of Hsp90-Cdc37-client protein targeted therapy. Furthermore, it is not likely that agents that target Hsp90Cdc37-client protein interaction will be used as monotherapy [56, 72, 78]. Rather, the known biological role and pre-clinical findings demonstrate that regulation of this complex will have more potential in the modulation of therapeutic response of other agents in certain cancers.

In conclusion, targeting Hsp90-Cdc37-client protein interaction is a reasonable and alternative strategy to the blockage of Hsp90 chaperone machinery and is a promising target for the cancer therapy.

\section{Additional file}

Additional file 1: The sup. Excel file for the comprehensive analysis of Hsp90-Cdc37's client proteins. Comprehensive Analysis of Hsp90-Cdc37's client proteins by using FunRich tool. (XLSX 313 kb)

\begin{abstract}
Abbreviations
AHA1: Activator of Hsp90 ATPase protein 1; AR: Androgen receptor;

Cdc37: Cell division cycle 37; CeCdc37: Cdc37 within Caenorhabditis elegans; CHIP: Carboxyl terminus of HSC70-interacting protein; CK2: Casein kinase 2; CYP40: Cyclophilin 40; FKBP5: FK506-binding protein 5; GM: Geldanamycin; HOP: Hsp70/Hsp90 organizing protein; Hsp90: Heat shock protein 90; PP5: Protein phosphatase 5; PPI: Protein-protein interaction; RIP1-3: Receptorinteracting protein kinases 1-3; SGTA: Small glutamine-rich TPR-containing protein alpha; S-TKc: Serine/threonine kinase catalytic domain; TAH1: TPR repeatcontaining protein associated with Hsp90; TTC4: Tetratricopeptide repeat domain 4; Unc45: Uncoordinated mutant number 45
\end{abstract}

\section{Funding}

This study was funded by grants from Science and Technology Development Fund, Macao S.A.R (FDCT) (024/2016/A1), Research Fund of University of Macau (MYRG2015-00091-ICMS-QRCM and MYRG2015-00101-ICMS-QRCM), and State Key Laboratory of Natural Medicines, China Pharmaceutical University (SKLNMKF201605).

Availability of data and materials

The material supporting the conclusion of this study has been included within the article.

\section{Authors' contributions}

TL drafted the manuscript. TL and JاJ participated in the discussion. All authors revised the manuscript. All authors read and approved the final manuscript.

Ethics approval and consent to participate

Not applicable

Competing interests

The authors declare that they have no competing interests. 


\section{Publisher's Note}

Springer Nature remains neutral with regard to jurisdictional claims in published maps and institutional affiliations.

\begin{abstract}
Author details
'State Key Laboratory of Quality Research in Chinese Medicine, Institute of Chinese Medical Sciences, University of Macau, Avenida da Universidade, Taipa, Macau, China. ${ }^{2}$ State Key Laboratory of Natural Medicines, China Pharmaceutical University, Nanjing, China. ${ }^{3}$ Department of Pathology, Xinxiang Medical University, 601 East Jinsui Ave, Xinxiang, Henan, China. ${ }^{4}$ Omigen, Inc., 15375 Barranca Pkwy, Irvine, CA H106, USA.
\end{abstract}

Received: 10 January 2018 Accepted: 20 April 2018 Published online: 27 April 2018

\section{References}

1. Whitesell L, Lindquist SL. HSP90 and the chaperoning of cancer. Nat Rev Cancer. 2005;5:761-72.

2. Schopf FH, Biebl MM, Buchner J. The HSP90 chaperone machinery. Nat Rev Mol Cell Biol. 2017;18:345-60.

3. Trepel J, Mollapour M, Giaccone G, Neckers L. Targeting the dynamic HSP90 complex in cancer. Nat Rev Cancer. 2010;10:537-49.

4. Zhao RM, Davey M, Hsu YC, Kaplanek P, Tong A, Parsons AB, et al. Navigating the chaperone network: an integrative map of physical and genetic interactions mediated by the Hsp90 chaperone. Cell. 2005;120:715-27.

5. Young JC, Agashe VR, Siegers K, Hartl FU. Pathways of chaperone-mediated protein folding in the cytosol. Nat Rev Mol Cell Bio. 2004;5:781-91.

6. Takayama S, Reed JC, Homma S. Heat-shock proteins as regulators of apoptosis. Oncogene. 2003;22:9041-7.

7. Wiech H, Buchner J, Zimmermann R, Jakob U. Hsp90 chaperones protein folding in vitro. Nature. 1992;358:169-70.

8. Chen B, Zhong DB, Monteiro A. Comparative genomics and evolution of the HSP90 family of genes across all kingdoms of organisms. BMC Genomics. 2006;7:156.

9. Harris SF, Shiau AK, Agard DA. The crystal structure of the carboxy-termina dimerization domain of htpG, the Escherichia coli Hsp90, reveals a potential substrate binding site. Structure. 2004;12:1087-97.

10. Karagoz GE, Duarte AMS, Ippel H, Uetrecht C, Sinnige T, van Rosmalen M, et al. N-terminal domain of human Hsp90 triggers binding to the cochaperone p23. P Natl Acad Sci USA. 2011;108:580-5.

11. Hessling M, Richter K, Buchner J. Dissection of the ATP-induced conformational cycle of the molecular chaperone Hsp90. Nat Struct Mol Biol. 2009;16:287-93.

12. Prodromou C, Roe SM, OBrien R, Ladbury JE, Piper PW, Pearl LH. Identification and structural characterization of the ATP/ADP-binding site in the Hsp90 molecular chaperone. Cell. 1997;90:65-75.

13. Panaretou B, Prodromou C, Roe SM, O'Brien R, Ladbury JE, Piper PW, et al. ATP binding and hydrolysis are essential to the function of the Hsp90 molecular chaperone in vivo. EMBO J. 1998;17:4829-36.

14. Roe SM, Prodromou C, O'Brien R, Ladbury JE, Piper PW, Pearl LH. Structural basis for inhibition of the Hsp90 molecular chaperone by the antitumor antibiotics radicicol and geldanamycin. J Med Chem. 1999;42:260-6.

15. Soga S, Shiotsu Y, Akinaga S, Sharma SV. Development of radicicol analogues. Curr Cancer Drug Targets. 2003;3:359-69.

16. Sharma SV, Agatsuma T, Nakano H. Targeting of the protein chaperone, HSP90, by the transformation suppressing agent, radicicol. Oncogene. 1998; 16:2639-45.

17. Chiosis G, Lucas B, Shtil A, Huezo H, Rosen N. Development of a purinescaffold novel class of Hsp90 binders that inhibit the proliferation of cancer cells and induce the degradation of Her2 tyrosine kinase. Bioorgan Med Chem. 2002:10:3555-64.

18. Pedersen KS, Kim GP, Foster NR, Wang-Gillam A, Erlichman C, McWilliams RR. Phase II trial of gemcitabine and tanespimycin (17AAG) in metastatic pancreatic cancer: a Mayo Clinic phase II consortium study. Invest New Drug. 2015;33:963-8

19. Acquaviva J, He SQ, Sang J, Smith DL, Sequeira M, Zhang CH, et al. mTOR inhibition potentiates HSP9O inhibitor activity via cessation of HSP synthesis. Mol Cancer Res. 2014;12:703-13.

20. Taipale M, Krykbaeva I, Koeva M, Kayatekin C, Westover KD, Karras Gl, et al. Quantitative analysis of Hsp90-client interactions reveals principles of substrate recognition. Cell. 2012;150:987-1001.
21. Wang L, Li L, Zhou ZH, Jiang ZY, You QD, Xu XL. Structure-based virtual screening and optimization of modulators targeting Hsp90-Cdc37 interaction. Eur J Med Chem. 2017;136:63-73.

22. Calderwood S. Cdc37 as a co-chaperone to Hsp90. Subcell Biochem. 2015; 78:103-12.

23. Gray PJ, Prince T, Cheng J, Stevenson MA, Calderwood SK. Targeting the oncogene and kinome chaperone CDC37. Nat Rev Cancer. 2008;8:491-5.

24. Siligardi G, Panaretou B, Meyer P, Singh S, Woolfson DN, Piper PW, et al. Regulation of Hsp90 ATPase activity by the co-chaperone Cdc37p/p50cdc37. J Biol Chem. 2002;277:20151-9.

25. Oppermann $\mathrm{H}$, Levinson W, Bishop JM. A cellular protein that associates with the transforming protein of Rous sarcoma virus is also a heat-shock protein. P Natl Acad Sci-Biol. 1981;78:1067-71.

26. Keramisanou D, Aboalroub A, Zhang ZM, Liu WJ, Marshall D, Diviney A, et al. Molecular mechanism of protein kinase recognition and sorting by the Hsp90 kinome-specific cochaperone Cdc37. Mol Cell. 2016;62:260-71.

27. Verba KA, Wang RYR, Arakawa A, Liu YX, Shirouzu M, Yokoyama S, et al. Atomic structure of Hsp90-Cdc37-Cdk4 reveals that Hsp90 traps and stabilizes an unfolded kinase. Science. 2016:352:1542-7.

28. Reed SI. The selection of S. cerevisiae mutants defective in the start event of cell division. Genetics. 1980:95:561-77.

29. Brugge JS, Darrow D. Rous sarcoma virus-induced phosphorylation of a 50,000-molecular weight cellular protein. Nature. 1982;295:250-3.

30. Eckl JM, Scherr MJ, Freiburger L, Daake MA, Sattler M, Richter K. Hsp90 center dot Cdc37 complexes with protein kinases form cooperatively with multiple distinct interaction sites. J Biol Chem. 2015;290:30843-54.

31. Cdc37 interacting proteins. https://www.picard.ch/downloads/Cdc37interactors.pdf. Accessed 8 Jan 2018.

32. Pathan M, Keerthikumar S, Ang CS, Gangoda L, Quek CYJ, Williamson NA, et al. FunRich: an open access standalone functional enrichment and interaction network analysis tool. Proteomics. 2015;15:2597-601.

33. Stelzl U, Worm U, Lalowski M, Haenig C, Brembeck FH, Goehler H, et al. A human protein-protein interaction network: a resource for annotating the proteome. Cell. 2005;122:957-68.

34. Ellebaek S, Brix S, Grandal M, Lantto J, Horak ID, Kragh M, et al. Pan-HERAn antibody mixture targeting EGFR, HER2 and HER3 abrogates preformed and ligand-induced EGFR homo- and heterodimers. Int J Cancer. 2016;139:2095105.

35. Freeman MR. HER2/HER3 heterodimers in prostate cancer: whither HER1/ EGFR? Cancer Cell. 2004:6:427-8.

36. Hutcheson LR, Barrow D, Hasmann M, Nicholson RI. Induction of erbB3/ EGFR heterodimers mediates resistance to pertuzumab in a tamoxifenresistant MCF-7 breast cancer cell line. Mol Cancer Ther. 2007:6:3373.

37. Massoner $\mathrm{P}$, Ladurner-Rennau M, Eder IE, Klocker H. Insulin-like growth factors and insulin control a multifunctional signalling network of significant importance in cancer. Brit J Cancer. 2010;103:1479-84.

38. Dziadziuszko R, Merrick DT, Witta SE, Mendoza AD, Szostakiewicz B, Szymanowska A, et al. Insulin-like growth factor receptor 1 (IGF1R) gene copy number is associated with survival in operable non-small-cell lung cancer: a comparison between IGF1R fluorescent in situ hybridization, protein expression, and mRNA expression. J Clin Oncol. 2010:28:2174-80.

39. Rushworth LK, Hindley AD, O'Neill E, Kolch W. Regulation and role of Raf-1/ B-Raf heterodimerization. Mol Cell Biol. 2006:26:2262-72.

40. Yuan WJ, Ding X, Wang Z, Yang BJ, Li XN, Zhang Y, et al. Two novel diterpenoid heterodimers, Bisebracteolasins A and B, from Euphorbia ebracteolata Hayata, and the cancer chemotherapeutic potential of Bisebracteolasin A. Sci Rep-Uk. 2017;7

41. Krogsgaard M, Li QJ, Sumen C, Huppa JB, Huse M, Davis MM. Agonist/ endogenous peptide-MHC heterodimers drive T cell activation and sensitivity. Nature. 2005:434:238-43.

42. AbdAlla S, Lother H, Quitterer U. AT(1)-receptor heterodimers show enhanced G-protein activation and altered receptor sequestration. Nature. 2000;407:94-8

43. Westin S, Kurokawa R, Nolte RT, Wisely GB, Mclnerney EM, Rose DW, et al. Interactions controlling the assembly of nuclear-receptor heterodimers and co-activators. Nature. 1998:395:199-202.

44. Neer EJ, Smith TF. G protein heterodimers: new structures propel new questions. Cell. 1996:84:175-8.

45. Mayer MP. Gymnastics of molecular chaperones. Mol Cell. 2010;39:321-31.

46. Gelis I, Keramisanou D, Aboalroub A. Protein kinase recognition and sorting by the HSP90 kinome-specific cochaperone CDC37. Biophys J. 2017;112:491. 
47. Shao JY, Prince T, Hartson SD, Matts RL. Phosphorylation of serine 13 is required for the proper function of the Hsp90 co-chaperone, Cdc37. J Biol Chem. 2003;278:38117-20.

48. Oberoi J, Dunn DM, Woodford MR, Mariotti L, Schulman J, Bourboulia D, et al. Structural and functional basis of protein phosphatase 5 substrate specificity. P Natl Acad Sci USA. 2016;113:9009-14.

49. Terasawa K, Yoshimatsu K, lemura S, Natsume T, Tanaka K, Minami Y. Cdc37 interacts with the glycine-rich loop of Hsp90 client kinases. Mol Cell Biol. 2006;26:3378-89.

50. Terasawa K, Minami Y. A client-binding site of Cdc37. FEBS J. 2005;272:4684-90

51. Prince T, Matts RL. Definition of protein kinase sequence motifs that trigger high affinity binding of Hsp90 and Cdc37. J Biol Chem. 2004;279:39975-81.

52. Shao JY, Irwin A, Hartson SD, Malts RL. Functional dissection of Cdc37: characterization of domain structure and amino acid residues critical for protein kinase binding. Biochemistry-Us. 2003;42:12577-88.

53. Eckl JM, Rutz DA, Haslbeck V, Zierer BK, Reinstein J, Richter K. Cdc37 (cell division cycle 37) restricts Hsp90 (heat shock protein 90) motility by interaction with $\mathrm{N}$ terminal and middle domain binding sites. J Biol Chem. 2013;288:16032-42.

54. Stepanova L, Yang G, DeMayo F, Wheeler TM, Finegold M, Thompson TC, et al. Induction of human Cdc37 in prostate cancer correlates with the ability of targeted Cdc37 expression to promote prostatic hyperplasia. Oncogene. 2000;19:2186-93.

55. Lei W, Mullen N, McCarthy S, Brann C, Richard P, Cormier J, et al. Heat-shock protein 90 (Hsp90) promotes opioid-induced anti-nociception by an ERK mitogen-activated protein kinase (MAPK) mechanism in mouse brain. J Biol Chem. 2017;292:10414-28.

56. Smith JR, Clarke PA, de Billy E, Workman P. Silencing the cochaperone CDC37 destabilizes kinase clients and sensitizes cancer cells to HSP90 inhibitors. Oncogene. 2009;28:157-69.

57. Gray PJ, Stevenson MA, Calderwood SK. Targeting Cdc37 inhibits multiple signaling pathways and induces growth arrest in prostate cancer cells. Cancer Res. 2007;67:11942-50.

58. Bartel DP. MicroRNAs: target recognition and regulatory functions. Cell. 2009:136:215-33.

59. Miyata Y, Nishida E. CK2 controls multiple protein kinases by phosphorylating a kinase-targeting molecular chaperone, Cdc37. Mol Cell Biol. 2004;24:4065-74.

60. Bandhakavi S, McCann RO, Hanna DE, Glover CVC. A positive feedback loop between protein kinase CKII and Cdc37 promotes the activity of multiple protein kinases. J Biol Chem. 2003;278:2829-36.

61. Miyata Y, Yahara I. The 90-kDa heat shock protein, Hsp90, binds and protects casein kinase-II from self-aggregation and enhances its kinase activity. J Biol Chem. 1992;267:7042-7.

62. Miyata Y, Yahara I. Interaction between casein kinase II and the 90-kDa stress protein, Hsp90. Biochemistry-Us. 1995;34:8123-9.

63. Shao J, Hartson SD, Matts RL. Evidence that protein phosphatase 5 functions to negatively modulate the maturation of the Hsp90-dependent heme-regulated elF2 alpha kinase. Biochemistry-Us. 2002;41:6770-9.

64. Chen YL, Hung MH, Chu PY, Chao TI, Tsai MH, Chen LJ, et al. Protein phosphatase 5 promotes hepatocarcinogenesis through interaction with AMP-activated protein kinase. Biochem Pharmacol. 2017;138:49-60.

65. Vaughan CK, Mollapour M, Smith JR, Truman A, Hu B, Good VM, et al. Hsp90-dependent activation of protein kinases is regulated by chaperonetargeted dephosphorylation of Cdc37. Mol Cell. 2008;31:886-95.

66. Cliff MJ, Harris R, Barford D, Ladbury JE, Williams MA. Conformational diversity in the TPR domain-mediated interaction of protein phosphatase 5 with Hsp90. Structure. 2006;14:415-26.

67. Polier S, Samant RS, Clarke PA, Workman P, Prodromou C, Pearl LH. ATPcompetitive inhibitors block protein kinase recruitment to the Hsp90-Cdc37 system. Nat Chem Biol. 2013;9:307.

68. Zhang T, Li YY, Yu YK, Zou P, Jiang YQ, Sun DX. Characterization of celastrol to inhibit Hsp90 and Cdc37 interaction. J Biol Chem. 2009;284: 35381-9.

69. Zhang T, Hamza A, Cao XH, Wang B, Yu SW, Zhan CG, et al. A novel Hsp90 inhibitor to disrupt Hsp90/Cdc37 complex against pancreatic cancer cells. Mol Cancer Ther. 2008;7:162-70.

70. Li YY, Karagoz GE, Seo YH, Zhang T, Jiang YQ, Yu YK, et al. Sulforaphane inhibits pancreatic cancer through disrupting Hsp90-p50(Cdc37) complex and direct interactions with amino acids residues of Hsp90. J Nutr Biochem. 2012;23:1617-26

71. Huang W, Ye M, Zhang LR, Wu QD, Zhang M, Xu JH, et al. FW-04-806 inhibits proliferation and induces apoptosis in human breast cancer cells by binding to N-terminus of Hsp90 and disrupting Hsp90-Cdc37 complex formation. Mol Cancer. 2014:13

72. Huang W, Wu QD, Zhang M, Kong YL, Cao PR, Zheng W, et al. Novel Hsp90 inhibitor FW-04-806 displays potent antitumor effects in HER2-positive breast cancer cells as a single agent or in combination with lapatinib. Cancer Lett. 2015;356:862-71.

73. Yu YK, Hamza A, Zhang T, Gu MC, Zou P, Newman B, et al. Withaferin A targets heat shock protein 90 in pancreatic cancer cells. Biochem Pharmacol. 2010;79:542-51.

74. Grover A, Shandilya A, Agrawal V, Pratik P, Bhasme D, Bisaria VS, et al. Hsp90/Cdc37 chaperone/co-chaperone complex, a novel junction anticancer target elucidated by the mode of action of herbal drug Withaferin A. BMC Bioinformatics. 2011;12

75. Gu MC, Yu YK, Gunaherath GMKB, Gunatilaka AAL, Li DP, Sun DX. Structureactivity relationship (SAR) of withanolides to inhibit Hsp90 for its activity in pancreatic cancer cells. Invest New Drug. 2014;32:68-74.

76. Li DR, Li C, Li L, Chen S, Wang L, Li Q, et al. Natural product Kongensin A is a non-canonical HSP90 inhibitor that blocks RIP3-dependent necroptosis. Cell Chem Biol. 2016;23:257-66.

77. Jacobsen AV, Silke J. The importance of being chaperoned: HSP90 and necroptosis. Cell Chem Biol. 2016;23:205-7.

78. Li T, Chen X, Dai XY, Wei B, Weng QJ, Chen XP, et al. Novel Hsp90 inhibitor platycodin D disrupts Hsp90/Cdc37 complex and enhances the anticancer effect of mTOR inhibitor. Toxicol Appl Pharm. 2017;330:65-73.

79. Li T, Chen X, Chen XP, Ma DL, Leung CH, Lu JJ. Platycodin D potentiates proliferation inhibition and apoptosis induction upon AKT inhibition via feedback blockade in non-small cell lung cancer cells. Sci Rep-Uk. 2016;6: 37997.

80. Wang L, Bao QC, Xu XL, Jiang F, Gu K, Jiang ZY, et al. Discovery and identification of Cdc37-derived peptides targeting the Hsp90-Cdc37 protein-protein interaction. RSC Adv. 2015;5:96138-45.

81. Rodina A, Wang T, Yan PR, Gomes ED, Dunphy MPS, Pillarsetty N, et al. The epichaperome is an integrated chaperome network that facilitates tumour survival. Nature. 2016;538:397

82. Caldas-Lopes E, Cerchietti L, Ahn JH, Clement CC, Robles Al, Rodina A, et al. Hsp90 inhibitor PU-H71, a multimodal inhibitor of malignancy, induces complete responses in triple-negative breast cancer models. P Natl Acad Sci USA. 2009;106:8368-73.

83. Heske CM, Mendoza A, Edessa LD, Baumgart JT, Lee SM, Trepel J, et al. STA8666, a novel HSP90 inhibitor/SN-38 drug conjugate, causes complete tumor regression in preclinical mouse models of pediatric sarcoma. Oncotarget. 2016; 7:65540-52.

84. Zhang YQ, Ng PKS, Kucherlapati M, Chen FJ, Liu YX, Tsang YH, et al. A pancancer proteogenomic atlas of PI3K/AKT/mTOR pathway alterations. Cancer Cell. 2017;31:820.

85. Martinelli E, Morgillo F, Troiani T, Ciardiello F. Cancer resistance to therapies against the EGFR-RAS-RAF pathway: the role of MEK. Cancer Treat Rev. 2017; 53:61-9.

86. Ibrahim SA, G adalla R, El-Ghonaimy EA, Samir O, Mohamed HT, Hassan H, et al. Syndecan-1 is a novel molecular marker for triple negative inflammatory breast cancer and modulates the cancer stem cell phenotype via the IL-6/STAT3, notch and EGFR signaling pathways. Mol Cancer. 2017; 16:57.

87. Li DR, Xu T, Cao Y, Wang HY, Li L, Chen S, et al. A cytosolic heat shock protein 90 and cochaperone CDC37 complex is required for RIP3 activation during necroptosis. P Natl Acad Sci USA. 2015;112:5017-22.

88. Yang $\mathrm{CK}$, He SD. Heat shock protein 90 regulates necroptosis by modulating multiple signaling effectors. Cell Death Dis. 2016;7:2126.

89. Foley C, Mitsiades N. Moving beyond the androgen receptor (AR): targeting AR-interacting proteins to treat prostate cancer. Horm Cancer-Us. 2016;7:84-103.

90. Xin Y, Huang M, Guo WW, Huang Q, Zhang LZ, Jiang G. Nano-based delivery of RNAi in cancer therapy. Mol Cancer. 2017;16:134.

91. Martins LR, Lucio P, Melao A, Antunes I, Cardoso BA, Stansfield R, et al. Activity of the clinical-stage CK2-specific inhibitor CX-4945 against chronic lymphocytic leukemia. Leukemia. 2014;28:179-82.

92. Wang L, Li L, Fu WT, Jiang ZY, You QD, Xu XL. Optimization and bioevaluation of Cdc37-derived peptides: an insight into Hsp90-Cdc37 protein-protein interaction modulators. Bioorgan Med Chem. 2017;25:233-40.

93. Zhao RL, Chen MJ, Jiang ZQ, Zhao FM, Xi BL, Zhang X, et al. Platycodin-D induced autophagy in non-small cell lung cancer cells via PI3K/Akt/mTOR and MAPK signaling pathways. J Cancer. 2015;6:623-31. 